\title{
A Narrative Review of the Renin-Angiotensin-Aldosterone System in the Placenta and Placental Bed of HIV Infected Women of African Ancestry with Preeclampsia
}

\author{
Shoohana Singh ${ }^{1} \cdot$ Jagidesa Moodley $^{2} \cdot$ Olive Pearl Khaliq $^{2} \cdot$ Thajasvarie Naicker $^{1}$ \\ Accepted: 15 July 2021 / Published online: 20 August 2021 \\ (C) The Author(s), under exclusive licence to Springer Science+Business Media, LLC, part of Springer Nature 2021
}

\begin{abstract}
Purpose of Review Both HIV infection and preeclampsia (PE), a pregnancy-specific disorder of hypertension and multi-system organ involvement, have high prevalence rates especially in low-to-middle-income countries. The immunoexpression of specific renin-angiotensin-aldosterone system (RAAS) receptors in the placenta and placental bed interface may forecast the risk of PE. Recent Findings Preeclampsia is a leading risk factor for mortality worldwide and remains a challenge in HIV-infected individuals especially those on antiretroviral therapy (ART). Irregular RAAS stimulation may be linked to the pathophysiology of hypertension in HIV infection and in PE. The AT1 receptor is expressed across all trimesters of pregnancy, within placental tissue, eliciting vasoconstriction. This increased expression is associated with the severity of PE, implying that the increased expression may be involved in the pathogenesis of this pregnancy disorder. The AT2 receptor expression in normotensive pregnancies was shown to be lower as compared to non-pregnant individuals. Furthermore, in the PE placental bed, the AT2 receptor is the predominant receptor subtype and is found in extravillous trophoblast cells where they facilitate vasodilation. However, AT4R in placentae of PE pregnancies are found to be significantly reduced compared to normotensives pregnancies. Summary The data on the role played by the RAAS pathway in pregnancy is conflicting. Investigation into a tissue-based RAAS with emphasis on immune-expression within the placenta and placental bed may help resolve this conundrum.
\end{abstract}

Keywords Renin-angiotensin-aldosterone system (RAAS) $\cdot$ Preeclampsia · Angiotensin II-type 1 receptor (ATR1) · Angiotensin II-type 2 receptor (ATR2) · Angiotensin II-type 4 receptor (ATR4)

\section{Background}

\section{Introduction}

In order to maintain a homeostatic environment, the reninangiotensin-aldosterone system (RAAS) functions as a crucial

This article is part of the Topical Collection on Preeclampsia

Shoohana Singh

singhs5@ukzn.ac.za

Thajasvarie Naicker

naickera@ukzn.ac.za

1 Optics and Imaging Centre, Doris Duke Medical Research Institute, School of Laboratory Medicine and Medical Sciences, College of Health Sciences, University of KwaZulu-Natal, Durban, South Africa

2 Department of Obstetrics and Gynaecology, Women's Health and HIV Research Group, School of Clinical Medicine, University of KwaZulu-Natal, Durban, South Africa blood volume and blood pressure regulator. This system is also an electrolyte controller and a systemic vasculature resistor that influences cardiac output and arterial pressure [1]. The main compounds in this multi-organ endocrine feedback system are renin, angiotensinogen (AGT), angiotensinconverting enzyme (ACE), angiotensin converting enzyme 2 (ACE2), angiotensin I (ANG I), angiotensin II (ANG II), and aldosterone. The liver, lungs and kidneys produce AGT, ACE and renin respectively while modulators of RAAS in the brain affect cognition [2]. The dysregulation of RAAS leads to vasoconstriction, cell proliferation, inflammation, and fibrosis $[3,4]$. Furthermore, the RAAS is implicated in the pathophysiology and progression of cardiovascular diseases, such as atherosclerosis and hypertension related pregnancy disorders [5].

Worldwide, approximately 800 women die each day from complications related to pregnancy and childbirth [6]. SubSaharan Africa (SSA) and Southern Asia account for $86 \%$ of these deaths with the former accounting for two-thirds of the total deaths $(196,000)$ [7]. The latest maternal mortality ratio 
(MMR) for South Africa (SA) is 119/100,000 live births, a dramatic improvement from 536/100,000 in 2016 [8]. Although, Human Immunodeficiency Virus (HIV) and Acquired Immunodeficiency Syndrome (AIDS) are the largest causes of maternal deaths in SA, epidemiological studies show that preeclampsia (PE), a pregnancy specific disorder is the main direct contributor of maternal mortality, accounting for $18 \%$ of all deaths [9-13]. Preeclampsia is a multi-system disorder characterized mainly by hypertension (systolic blood pressure $\geq 140 \mathrm{mmHg}$ or diastolic pressure $\geq 90 \mathrm{mmHg}$ ). One or more of the following clinical signs may be involved: proteinuria ( $\geq 0.3$ obtained from a 24 -hour urine test or a $1+$ to $2+$ on a urine dipstick), utero-placental dysfunction and maternal organ dysfunction (kidney, liver and hemostatic system) [14, 15].

Sub-Saharan Africa has the highest global burden of HIV infection. In 2020, the overall prevalence of HIV infection in SA was $13 \%$ [16]. Unfortunately, women are disproportionately affected; $26 \%$ of women are HIV infected compared to $15 \%$ of men [17]. The National Department of Health Antenatal HIV and Syphilis surveillance study found that the HIV infection rate among pregnant women was $17 \%$ [18]. South Africa is therefore faced with both high HIV infection and PE prevalence rates $[18,19]$. The coinfection of $\mathrm{PE}$ and HIV remains a concern to the South African healthcare system. Moreover, the data on the association between HIV infection and PE is conflicting because of opposing immune responses. While pregnant women receiving highly active antiretroviral treatment (HAART) have an increased risk for PE development due to immune reconstitution, other reports suggest no change in the frequency of $\mathrm{PE}$ when both conditions co-exist [20].

In this review, we explore the role of RAAS in uteroplacental tissue of pregnancy, drawing attention to novel combined effects of HIV infection and PE. Understanding the mechanisms of RAAS at the placenta and placental bed level of HIV infected women of African ancestry is important for two reasons. Firstly, increased knowledge of the mechanisms in which RAAS contributes to the clinical condition of both mother and fetus is critical to improve public health efforts to prevent hypertension, cardiovascular disease and premature mortality. Secondly, the study of RAAS-specific pathophysiology linked to PE may reveal important immunologic and inflammatory pathways that could affect susceptibility to the development of these high-risk pregnancies.

At present, there is a paucity of information on RAAS in the placental bed particularly in women with both HIV infection and PE. This review will focus on signal transduction at the placenta and placental bed in HIV infected women with PE. More specifically, it will examine angiotensin II-type 1 receptor (ATR1), angiotensin II-type 2 receptor (ATR2), and angiotensin II-type 4 receptor (ATR4) for greater understanding of RAAS. Unraveling this pathway will aid in the development of new preventative and therapeutic interventions for women of African ancestry who have concurrent HIV infection and PE.

\section{The Role of Renin-Angiotensin-Aldosterone System (RAAS)}

Renin (an aspartyl proteolytic enzyme) is primarily released from juxtaglomerular cells (JGC) of the afferent arteriole in the glomeruli of the kidney. Renin release is stimulated by either renal artery hypotension, decreased sodium delivery to the renal tubules or sympathetic nerve activation (via the $\beta 1$ adrenoceptor) [21].

When renin is released into blood, it catalyzes the conversion of angiotensinogen by cleaving it to form the decapeptide, angiotensin I. Angiotensinogen is an $\alpha 2$ globulin precursor of angiotensin, and a hormone produced in the liver, kidney, adrenal glands, brain, heart and blood vessels, and adipose tissues [22]. The lungs' vascular endothelium produces the angiotensin-converting enzyme (ACE), which cleaves angiotensin I to form the potent octapeptide, angiotensin II [23]. Angiotensin-converting enzyme 2 (ACE2) catalyzes the hydrolysis of angiotensin II (a vasoconstrictor peptide) into angiotensin (1-7) (a vasodilator), thereby lowering blood pressure [24]. The ACE2 has gained much attention recently due to the coronavirus disease of 2019 (COVID-19) pandemic as it serves as the entry receptor for the virus, severe acute respiratory syndrome coronavirus-2 (SARS-CoV-2).

\section{Renin-Angiotensin-Aldosterone System Signal Transduction}

The RAAS depends on several different receptors for signal transduction. Widely researched receptors for signal transduction of ANGII, occurs via two main receptor types, Angiotensin II-type 1 receptor (AT1R) and Angiotensin IItype 2 receptor (AT2R). Both AT1 and AT2 receptors belong to the seven transmembrane G-protein coupled receptors and have an equivalent affinity to ANG II [25].

Angiotensin II-type 1 receptor (AT1R) is located in the renal vasculature, glomerular mesangium, interstitial cells and proximal tubules, lung, liver, brain, kidney, adrenal gland, skin, and endometrium. It functions in a signaling pathway to increase intracellular calcium absorption. Moreover, it is involved in vasoconstriction, sympathetic activity (release of noradrenaline from sympathetic nerve terminals), amplifying vasoconstriction and increasing the rate and force of contraction of the heart [26]. The AT1 receptor also causes proximal tubular reabsorption of sodium ions, secretion of aldosterone from the adrenal cortex, and cell growth in the cardiac left ventricle and in the arterial wall [26]. 
Angiotensin II via the AT1R controls cellular growth, adhesion, migration, and intercellular matrix deposition, influencing chronic adaptive changes in vascular and cardiac growth, remodeling, repair, and atherosclerosis. In vascular smooth muscle cells and endothelial cells, ANG II acting via the AT1R stimulates phospholipase A2 activity, leading to the release of arachidonic acid and eicosanoids, which influence vascular and renal mechanisms that are important in the regulation of blood pressure and cell growth [22].

The AT1 receptor promotes intracellular signaling pathways through the activation of various protein kinases, subunits of nicotinamide adenine dinucleotide phosphate (NADPH) oxidase, and growth factor receptor transactivation. Alternately signaling is mediated by direct interactions with AT1R proteins such as Janus kinase 2 (JAK2), phospholipase C (PLC) $\gamma 1$, AT1 receptor associated protein (ATRAP), type 1 angiotensin II receptor-associated protein (ARAP1) and Guanine nucleotide exchange factor (GEF)-like protein (GLP) [27].

The expression of AT1 receptors is variable during pathophysiological conditions including, reno-vascular hypertension, myocardial infarction, ventricular hypertrophy, and bilateral nephrectomy [28]. Overexpression of the vascular AT1 receptor occurs in hypercholesterolemic patients. These findings may help to explain why hypercholesterolemia is frequently associated with hypertension and why blockade of the RAAS attenuates the progression of atherosclerosis (Figure 1) [30]. The down-regulation of AT1 receptors in sepsis is the main reason for the weakened responsiveness of blood pressure and of aldosterone formation to ANG II and, therefore, may contribute to the characteristic septic shock [31]. AT1 receptors are found throughout life in all tissues involved in the cardiovascular activity of Ang II [32, 33].

Fig. 1 Diagram illustrating the activation pathway of AT1R and pathophysiology of overexpression of this receptor (adapted from Fanelli et al., 2011 [29])
The AT1 receptor is expressed across all trimesters of pregnancy occurring within placental syncytiotrophoblast, cytotrophoblast, Hofbauer cells, and the fetal vascular endothelium [34]. Notably, majority of the components of the RAAS are expressed in the placenta from early gestation onwards, suggesting that the RAAS may have a role in the human placenta throughout gestation [34].

Another study demonstrated significant increase of AT1 receptor expression within human placental syncytiotrophoblast cells and villous endothelium in pregnancy-induced hypertension (PIH) [35]. This expression increased with the severity of PIH, implying that the increased expression may be involved in the pathogenesis of PIH [35]. The increased expression of AT1 receptor further supports the hypothesis that a tissue-based RAAS exists where pro-renin and its receptor act, potentially a novel pathway to understanding the role of RAAS in the pathogenesis of preeclampsia [36].

Angiotensin II-type 2 receptor (AT2R) is located in renal vessels, glomeruli and tubules, and when activated inhibit cell growth, initiate apoptosis, cause vasodilation and promote fetal tissue development (Figure 2). The AT2 receptors are upregulated in various fetal tissue (kidney and intestine) suggesting a possible role in modulation of growth and remodeling of fetal vasculature [38]. However, their level of expression declines remarkably after birth: in adults, they are found only in a few organs, including the brain, adrenal gland, heart, and myometrium with lower levels in fallopian tube. Post injury and during wound healing, AT2 receptor expression increases in limited tissues, including the heart, vasculature, and kidney $[32,33]$.

Angiotensin II- type 3 receptor (AT3R) demonstrates unusual pharmacological action and interactions, with limited gene information in humans. The AT3 receptor has been

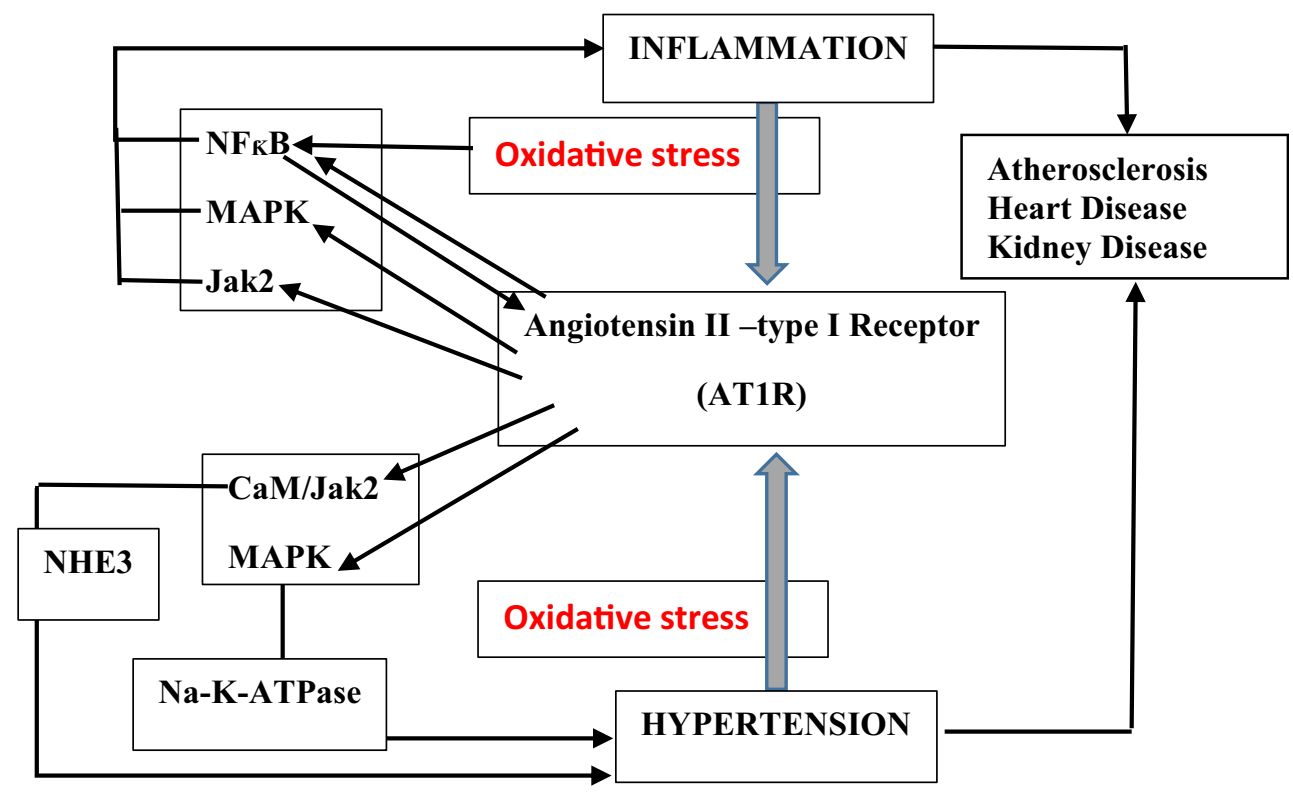


Fig. 2 Diagram illustrating the cellular signaling pathway AT2R and pathophysiology of overexpression of this receptor (adapted from Hatip-Al-Khatib, I, et al.) [37]). ANP, atrial natriuretic peptide; $\mathrm{COX} 2$, cyclooxygenase 2 ; MAPK, mitogen-activated protein kinase; Mrg-D, Mas-related G-protein coupled receptor membrane $\mathrm{D}$; PTP, protein phosphatase; + and stand for stimulation and inhibition, respectively

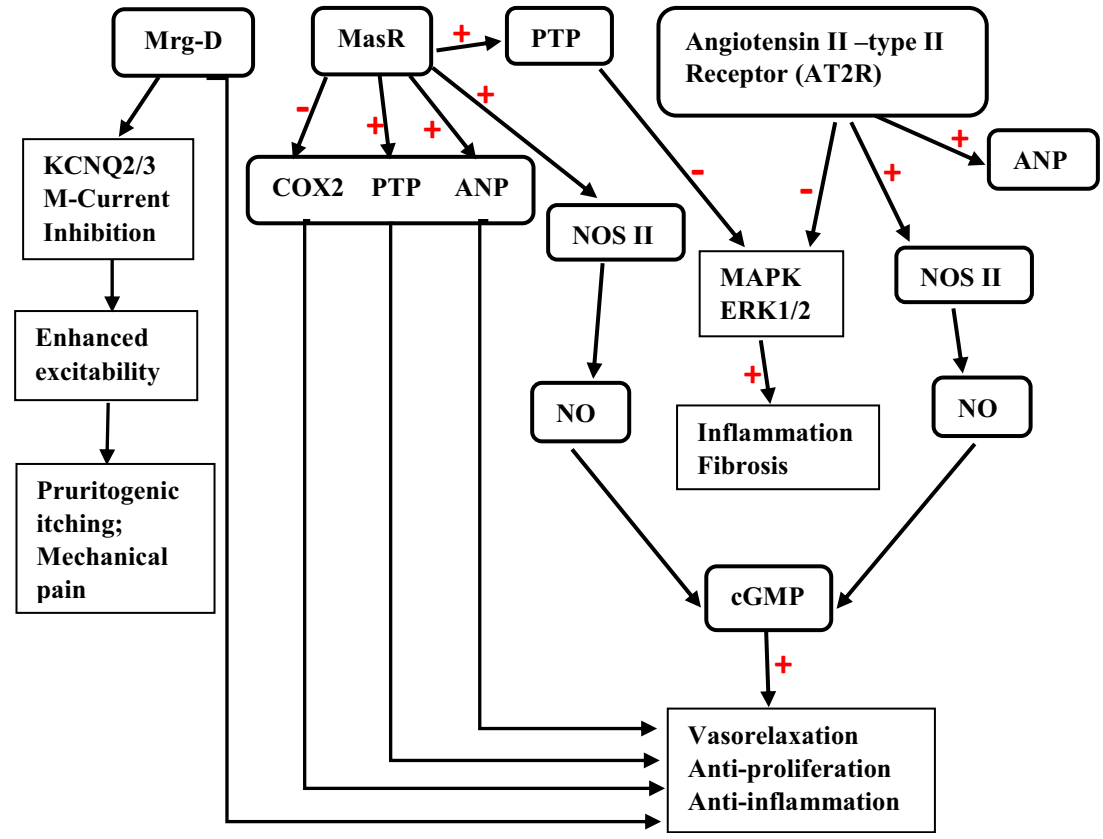

identified in animal studies and shown to be a smooth muscle receptor [39].

Angiotensin II- type 4 receptor (AT4R) has a high affinity for membrane binding sites for [125I] Ang IV peptide. The AT4R receptors occur predominately in the brain and to some extent in heart, kidney, adrenal gland, and blood vessels. The AT4R receptor is believed to be associated memory, regulation of blood flow and vasodilation [40, 41]. There are limited reports on AT4R receptors in humans, but extensive mammalian studies show that these receptors are not serpentine $G$ protein-linked but rather an insulin-regulated aminopeptidase (IRAP), a membrane-associated aminopeptidase that codistributes with the GLUT4 transporter (Figure 3) [42]. Furthermore, AT4 receptors have been found in endothelial and smooth muscle cells and most importantly, in extravillous trophoblast cells where they facilitate vasodilation synergistically with nitric oxide [43••]. Williams et al. (2010) investigated the expression of AT4R in placentae of PE pregnancies and found a significant reduction of AT4R at protein and mRNA level in PE compared to normotensives which showed high levels throughout pregnancy [43••]. The study also revealed that AT4R staining of extravillous trophoblast cells in early pregnancy showed higher AT4R levels implicating their critical role in placentation and concluded that the contrary may result in the development of PE [43••].

In an attempt to quantify levels of AT4R in plasma, Khaliq et al. (2020) measured the concentration of plasma soluble angiotensin IV receptor (sAT-4) on healthy normotensive and PE pregnancies. They showed that plasma circulating levels of sAT-4 in women with severe features of PE (identified as systolic blood pressure $\geq 160 \mathrm{mmHg}$ and/or diastolic blood pressure $\geq 110 \mathrm{mmHg}$, with proteinuria $\geq 2.0 \mathrm{~g}$ in a $24-\mathrm{h}$ urine test or $\geq 3+$ on a dipstick) had lower levels than normotensives and those with PE without severe features [44••].

MAS receptor is the main receptor for RAAS produced angiotensin-1-7 [Ang(1-7)] with a similar structure to other G-protein coupled receptors (Figure 2) [41]. The MAS receptor when activated by binding Ang (1-7) counteracts several effects of angiotensin-II activated angiotensin receptors. Consequently, MAS receptor agonists have similar therapeutic effects as angiotensin-II receptor antagonists which include lowering blood pressure by promoting vasodilation [45]. Expressed on the endothelium, the MAS receptor binds with Ang(1-7) further altering local redox balance, oxidative stress reduction and anti-fibrosis [46]. The MAS receptors are also expressed in the brain with moderate amounts in the heart, kidney and vessels [46]. The heptapeptide Ang (1-7) elicits a nitric oxide (NO) dependent vasorelaxation via the MAS receptor [47]. This evidence further supports the theory of the two main axes of RAAS, which oppose each other to maintain vascular homeostasis. This includes the classical vasoconstrictive axis, renin/ACE/ANGII/AT1R, and the opposing vasorelaxant axis, ACE2/Ang(1-7)/MAS receptor [48, 49].

There are two main axes of the renin-angiotensin-aldosterone system that counteract each other in terms of vascular control, as illustrated in Figure 4.

\section{Renin-Angiotensin-Aldosterone System Activation in Pregnancy}

During a normotensive pregnancy, the RAAS undergoes changes to maintain a delicate balance for both mother and fetus as dual contributors to the signaling cascade. There is a generalized increase in renin and angiotensinogen, resulting in 


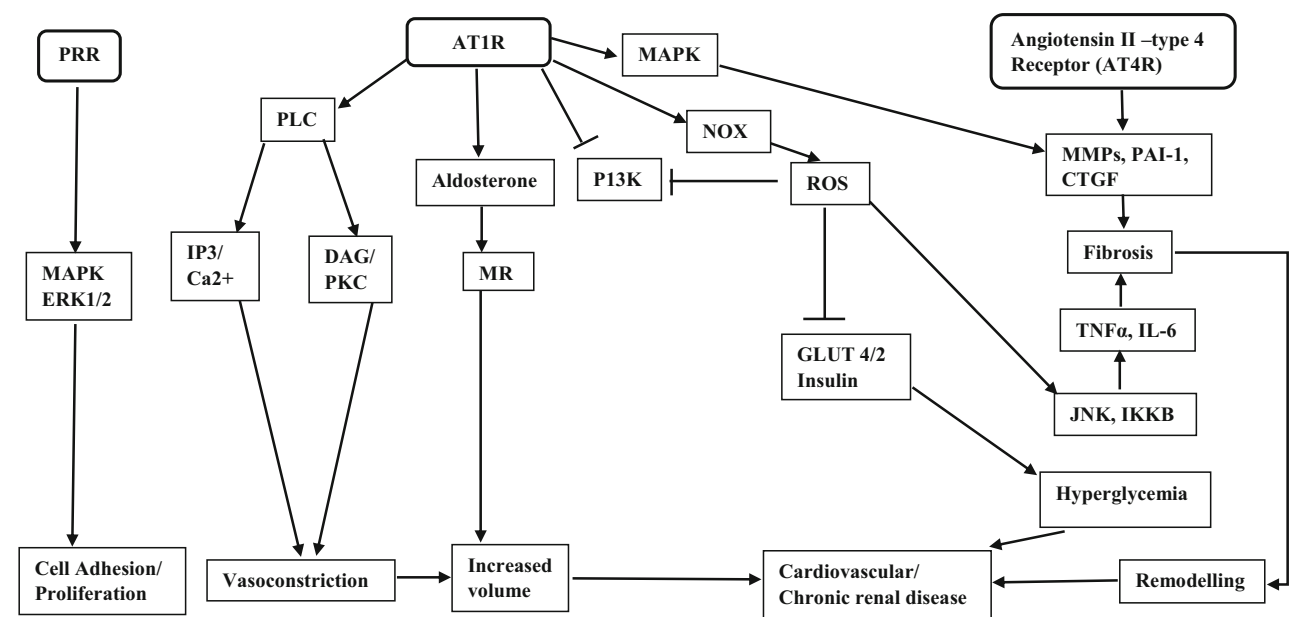

Fig. 3 Diagram illustrating the cellular signaling pathways of AT4R and pathophysiology of overexpression of this receptor. AT1R inhibits ACE2, but increases ADAM (A Disintegrin and Metalloproteinase). CTGF, connective tissue growth factor; DAG, diacyl glycerol; Glut, glucose transporter; IKK- $\beta$, inhibitor of nuclear factor-kappa-B kinase; IP3, inositol triphosphate; JNK, c-Jun N-terminal kinases; MAPK, mitogen-activated protein kinase; MMPs, matrix metalloproteinases; MR, mineralocorticoid receptor; NOS, nitric oxide synthase; NOX, NADPH oxidase; PAI-1, plasminogen activator inhibitor-1; PI3K,

increased ANG II and aldosterone levels. This is a compensatory mechanism to achieve the same vasomotor response as a phosphoinositide 3-kinase; PKC, protein kinase C; PLC, phospho lipase $C$; PRR, prorenin receptor; ROS, reactive oxygen species; TNF- $\alpha$, tumor necrosis factor alpha (adapted from Hatip-Al-Khatib, I, et al., [37]). Ang II acts directly on vascular smooth muscle as a powerful vasoconstrictor and is able to alter renal sodium and water absorption through its ability to stimulate the zona glomerulosa cells of the adrenal cortex to synthesize and secrete aldosterone. Ang II stimulates aldosterone release, followed by fluid and salt retention and subsequent volume increase.

non-pregnant woman. The only RAAS compound that is decreased during pregnancy is ACE [51•]. Also there is an

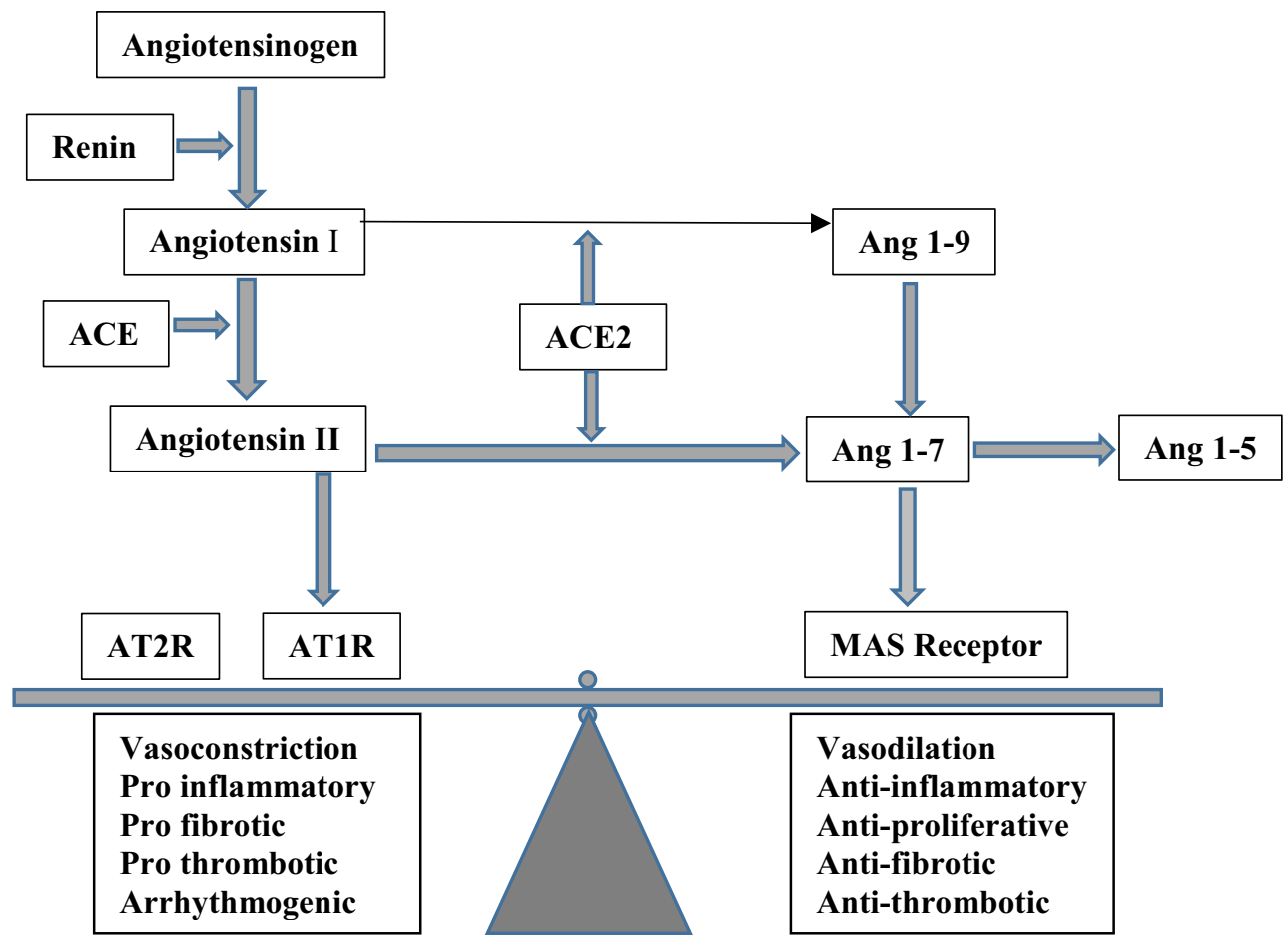

Fig. 4 The RAAS system, adapted from Lubel et al. [50], has become increasingly complex with alternative ways of angiotensin II (AngII) formation besides angiotensin-converting enzyme (ACE) and a second form of ACE (ACE2), angiotensin(1-9), and angiotensin(1-7). Clinically important could be that Angiotensin II can also bind to AngII type 2
(AT2) receptors stimulating a greater degree of vasoconstriction, proinflammatory compounds, prothrombotic pathways, and arrhythmogenic effects, whereas Ang1-9 and Ang1-7 bind to MAS receptors to elicit vasodilation with overall antiproliferative, antiinflammatory, antifibrotic, and antithrombotic effects. 
increased level of plasma Ang-(1-7) in normotensive pregnancies compared to non-pregnant individuals in contrast to decreased Ang-(1-7) in PE compared to normotensive pregnancies [52]. This indicates that in PE, the decreased plasma Ang-(1-7) in the presence of elevated Ang II is consistent with the development of hypertension [53].

Apart from the typical circulating RAAS compounds (normally involving kidneys and lungs); evidence shows a production of RAAS compounds from other tissues like the brain, ovary and placenta. Placental tissue expresses pro-renin, renin, ACE, ANGI, ANG II, and the AT1R receptor [51, 54]. A study on the corpus luteum (CL) of the ovary was conducted to measure levels of the inactive form of renin, pro-renin; the authors found that pro-renin and renin were lower in the absence of a CL and in particular pregnancies conceived in the absence of a CL were more disposed to develop PE [55••].

\section{RAAS Activation in the Placenta and Placental Bed of Women with Preeclampsia}

The exact etiology of PE has not yet been unraveled; but the placenta is generally regarded as a role player in the pathophysiology. This is based fact that PE can occur only in the presence of a pregnancy and resolves after delivery of the placenta. Histopathological findings indicate that PE is two phased. The first phase is the failure in vascular remodeling of spiral arteries into wide-bore channels within the placental bed $[56,57]$. This failure causes reduction of blood flow to the placenta, thus initiating the second phase. Placental tissue hypoxia, results in trophoblastic debris, necrotic tissue and a cascading release of biomarkers such as soluble fms-like tyrosine kinase (sFlt-1) and soluble endoglin (sEng) into general circulation $[56,57]$.

During PE, some evidence suggests that ACE levels remain relatively stable while the vasodilator, ANG (1-7) is substantially reduced [51•]. However, elevated levels of ACE-2 and Ang (1-7) in PE have also been reported, implying that the local expression of Ang-(1-7) and ACE2 may have a principal role in the placenta, differing from the regulation of the circulating RAAS [58•]. Another study showed AT1R expression did not vary with gestation and the highest levels of AT2R expression was found in early and mid-termination of pregnancy placentae compared to term in normal versus PE pregnancies [43••]. In contrast, expression of AT4R is significantly reduced in PE compared to normotensive pregnancies [43••]. Moreover, in PE, it was found that the AT1 receptor gene was 5-fold upregulated within the decidua with a significant increase in an angiotensin II type I agonistic autoantibody (AT1-AA) in both mother and infant [59•]. Gene expressions for renin, ACE, and angiotensinogen are higher in the placental bed decidua than placenta in both normotensive and PE, whereas the expression of AT1R is 10-fold higher in placenta compared to decidua in both groups [59•].

A study on placental chorionic villi showed ANGII immunoexpression was significantly higher in PE pregnancies, with no difference in Ang (1-7) levels. Angiotensinogen and AT1 receptor gene expression was significantly higher in PE; however, no differences were observed in renin, ACE, ACE-2, or neprilysin gene expression. The MAS receptor (involved in vasodilation) mRNA in PE patients was decreased with AT1 receptor being the predominant receptor subtype in normotensive and PE chorionic villi. These results suggest that the elevated angiotensin II, acting through the AT1 receptor, may facilitate vasoconstriction in placental chorionic villi and contribute to impaired fetal blood flow and decreased fetal nutrition observed during PE [60••].

A similar study of uterine placental bed from normotensive pregnancies, demonstrated that angiotensin II, angiotensinogen, ACE, AT1, AT2, and MAS receptor immunoexpression were lower in comparison to nonpregnant participants [61]. In preeclamptic uterine placental bed, angiotensin II, renin, ACE mRNA expression were significantly enhanced than normotensive pregnancies. The AT2 receptor was the predominant receptor subtype in the nonpregnant fundus, whereas all angiotensin receptor binding was undetectable within normotensive pregnant and preeclamptic uterine placental bed compared with non-pregnant fundus. These authors' findings imply that the maternal uterine placental bed may play an endocrine role by producing AngII, which acts in the adjacent placenta to constrict fetal chorionic villi vessels where AT1 receptors predominate, implicating decreased maternal-fetal oxygen exchange and fetal nutrition, a known characteristic of PE development [61].

Another study, showed no variation of placental AT1R expression with gestation. An extremely elevated level of AT2R expression was demonstrated in early and midtermination of pregnancy (TOP) placentae compared to term pregnancy. Expression of AT4R was increased in term, with a significant reduction in PE placentae. Additionally, a culture with AngIV or AngII increased extravillous trophoblast (EVT) invasion from placental explants, corroborating elevated trophoblast proliferation and reduced apoptosis. Expression of AT1R, AT2R and AT4R was also characterized in human placenta throughout normal pregnancy and in PE. The latter study highlights an important role for both AngIV and AngII in normal pregnancy [43••]. As noted in Table 1, several studies have attempted to address the association of RAAS with PE using either genotyping or immunohistochemistry analysis. Nonetheless, there are limited studies on uteroplacental tissue.

Some epidemiological studies which have investigated AT1 and AT2 receptor gene polymorphisms in PE and normal pregnancy based on ethnicity showed that the frequency of the AT2R-GG genotype in the PE group was significantly greater 


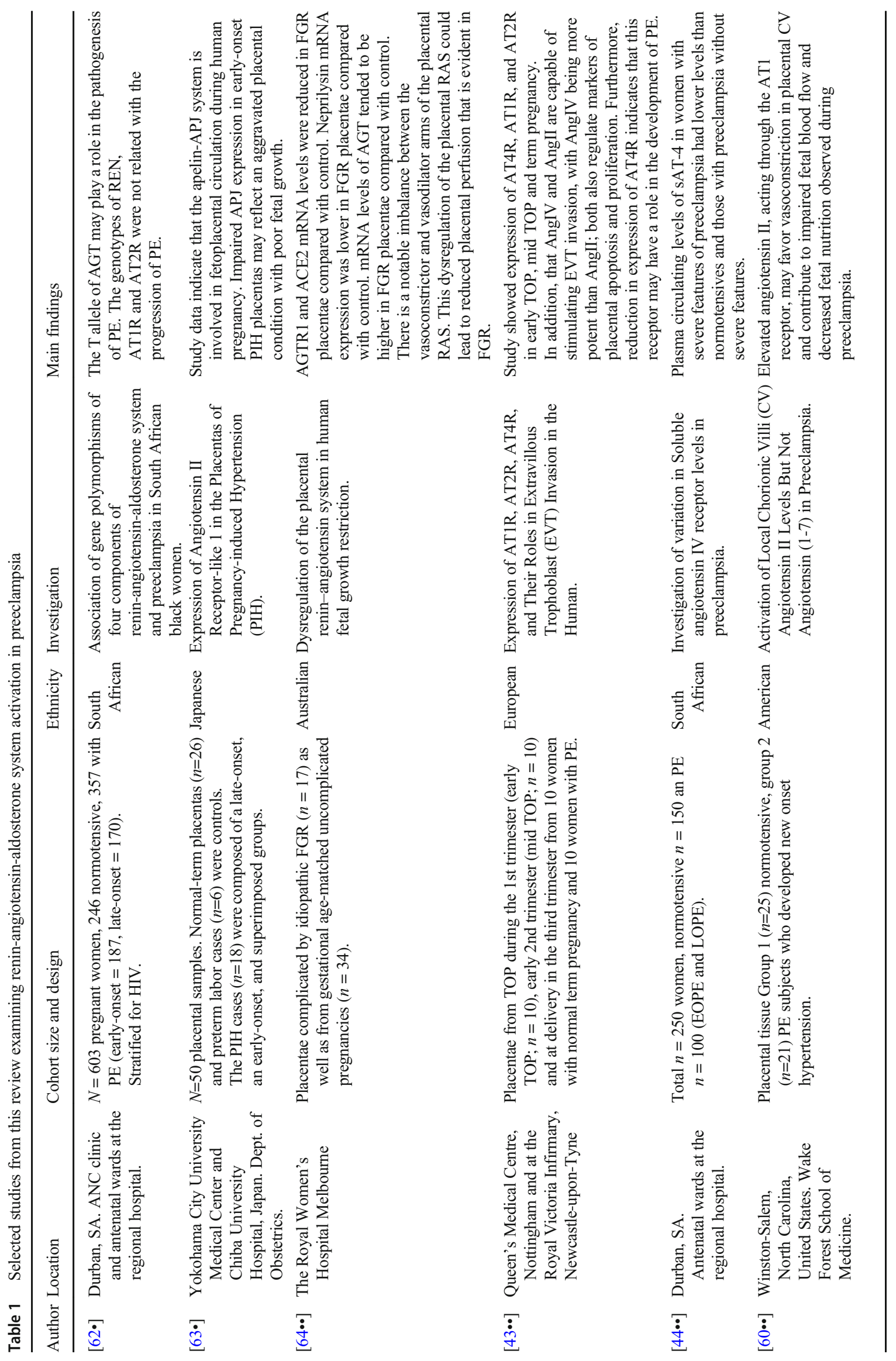


than in controls for Afro-Caribbean women (49.3\% vs $26.9 \%$, $p=0.004)$, compared to an absence of a frequency difference in Asian and Caucasian women [65]. These findings are supported by another American study that spanned a 10-year period and collectively found that the rate of diagnoses for all hypertensive disorders combined and for PE individually were highest among African-American women across all regions regardless of economic status. Although hospitalization rates for PE decreased over time for most groups, differences in rates between Caucasians and African-American women increased over the 10-year period. African-American and Hispanic women were more likely than Caucasian women to have diabetes and were at higher risk of PE development; the PE prevalence rates were higher in these groups both with and without diabetes than in corresponding groups of Caucasian women [66].

Preeclampsia fosters the AT1-AA that stimulates the AT1 receptor [67]. The AT1-AA binds the AT1 receptor, leading to stimulation of pro-inflammatory transcription factors such as Tumor necrosis factor alpha (TNF- $\alpha$ ) and reactive oxygen species (ROS) [68]. Furthermore, AT1-AA/AT1R binding promotes an upregulation of powerful vasoconstrictors such as ET-1, sFlt-1, and sEng thereby dysregulating RAAS [69]. This suggests that the production of an autoimmune antibody against AT1 receptor may contribute to the pathogenesis of PE. Subsequent studies showed that AT1-AA obtained from women with PE activates NADPH oxidase in vascular smooth muscle and trophoblast cells, implying that AT1-AA is a major mediator that increases oxidative stress, a pathologic phenotype found in placentas of PE women [70]. Another supportive study verified the presence of AT1-AA in patients with $\mathrm{PE}$ and showed that it induces plasminogen activator inhibitor-1 production [71]. They also showed that AT1-AA mobilizes intracellular calcium, activated the nuclear factor of activated $T$ cells and increased interleukin- 6 and plasminogen activator inhibitor-1 secretion from mesangial cells at a significantly higher level than that achieved with Immunoglobulin $\mathrm{G}$ (IgG) from normotensive patients. [72]. The data from these researchers imply that the AT1-AA may be responsible for the vascular lesions in PE [73]. Moreover, in another study over $80 \%$ of PE patients were AT1-AA positive, whereas less than $6 \%$ normotensive pregnancy were AT1-AA positive, confirming that a high prevalence of AT1-AA is associated with PE development [74]; these authors were also able to provide evidence that AT1-AA isolated from PE patients caused significant vasoconstriction in conducting arteries as well as lower resistance arteries. Apart from this major breakthrough, the authors also conclusively demonstrated that AT1AA induces vasoconstriction via activation of AT1 receptor [74]. Collectively, this research implies that the adherence of AT1-AA to the AT1 receptor may result in characteristics of $\mathrm{PE}$, such as increased peripheral vascular resistance, elevated cardiac afterload, promoted cardiac hypertrophy and decreased blood supply.

\section{Preeclampsia and HIV}

The established list of risk factors for PE include primiparity, previous $\mathrm{PE}$, family history of $\mathrm{PE}$, high maternal prepregnancy body mass index, ethnicity where women of African Ancestry are at higher risk, and underlying medical conditions such as diabetes mellitus, antiphospholipid antibodies, autoimmune, or renal disease and chronic hypertension [75]. Another risk factor is HIV infection. A 26-year population-based retrospective study has shown that pregnant women who are HIV positive were significantly more prone to PE and more so on receipt of HAART [76, 77]. However, this remains a debatable issue as there are conflicting studies on the risk of PE development [20, 78]

In SA, KwaZulu-Natal is the most severely affected province with just over 40\% HIV prevalence [79]. The standard HAART and Prevention of Mother to Child Transmission (PMTCT) program has drastically reduced transmission of HIV from mother to child to $3.8 \%$ however, not without its own complications [17].

A retrospective study in regional and tertiary hospitals of SA evaluated the rate of HIV/AIDS infection in women with PE. It was one of the first studies to report the rate of HIV infection in women with preeclampsia in comparison with a control group without preeclampsia and found that the rate of HIV/AIDS was significantly lower in women with preeclampsia $[80,81]$. The mechanism behind this outcome has not been extensively researched but is believed that HIV has a protective function either via immune suppression or the HIV tat protein strongly mimics vascular endothelial growth factor (VEGF), it is plausible to assume that HIV infection may enhance the angiogenic imbalance in PE [82]. Our research team performed a small-scale study on soluble E-selectin (a circulating adhesion molecule expressed on endothelial cells, activated by inflammation or stress) demonstrating an elevation of sE-selectin in preeclamptic HIV uninfected compared to the normotensive HIV uninfected group. However, when stratified by HIV status, there was no substantial difference observed between preeclamptic HIV infected and normotensive HIV infected groups [83]. We also showed significant elevation of another complement component, adipisin (complement factor $\mathrm{D}$ that is a serine protease synthesized by adipocytes and this enzyme activates the alternative pathway of the complement, triggering the natural defense against infections) between HIV uninfected normotensive vs HIV uninfected PE as well as a difference between HIV uninfected PE vs HIV infected PE [84]. This supports the theory that immune suppressive action of HIV is beneficial in reducing risk of PE. A prevalence study in Italy, of African-American Black ethnic group demonstrated that pregnant women with 
HIV infection seem to be protected against gestational hypertension and $\mathrm{PE}$ based on clinical presentation. Inferring that a lowered immune response at conception of the HIV infected women could account for unopposed trophoblast invasion resulting in improved placentation [85]. Whereas, at the University of Gondar specialized referral Hospital, the maternal HIV infection, PE, and prematurity were associated with low birth weight deliveries [86].

Conflicting results from a Soweto cohort of 2600 pregnant woman showed no significant difference between risk of developing PE due to HIV seropositivity, also based on clinical presentation [87]. This confliction may possibly be due to the administration of HAART. There are several studies claiming that HAART increases the risk of PE development, like the study in Western Cape, SA where extremely low birth weight infants were delivered due to hypertension related disorder, PE being the main cause. Although HIV infection showed no association, mothers who were on 4 weeks of anti-retroviral therapy were more likely to develop severe PE [88]. This was further supported by a secondary analysis examination of institutional maternal-deaths data presented in the Saving Mothers report 2014, which records maternal deaths in the nine provinces of SA. The secondary analysis showed that although HIV and AIDS remains the primary cause of death, it was still associated with decreased risk of HDP; use of HAART increased this risk [89]. Another elaborate review highlighted the crucial role that biomarkers and immune cells including, natural killer (NK) cells, cytokines and human leukocyte antigen (HLA-G) play in predisposing HIV-infected women who are on HAART into developing PE. That review provided evidence of PE being less common in HIV infected treatment naïve pregnancies and that data showed the risk of PE development in HIV infected women is greater in those receiving HAART. However, there are substantial unknown areas of research involving these immune markers role in the development of PE in HIV associated pregnancies [90]. A systematic review of 21,200 women in 13 different studies stated differing conclusions that there was insufficient evidence to infer that women living with HIV receiving either HAART or ART monotherapy are at increased risk to $\mathrm{PE}$ as pregnant women without HIV infection [20]. Bearing in mind that a pivotal comparison was not drawn against HIV infected pre-eclamptics not on HAART treatment.

\section{The Role of RAAS in the Placenta and Placental Bed of HIV Infected Pregnancies}

Hypertension has been the leading risk factor for mortality worldwide and remains a challenge in HIV infection especially those on ART [91]. Irregular RAAS stimulation may be linked to the pathophysiology of hypertension and insulin resistance in HIV infection [92]. It is now known that HIV infected individuals present with elevated plasma renin activity as a result of increased RAAS upstream activation [93].
This anomaly is elaborated by the similarity between the structure of HIV-1 protease and that of renin [94]. An increased renin production in immune cells occurs due the presence of HIV, thereby activating RAAS. Furthermore, renin contributes to HIV replication via the renin signaling cascade and through cleavage of the HIV Gag polyproteins [92]. The authors also found that the viral capsid (core) p24 protein was significantly increased in HIV infected cells incubated with renin. Apart from abnormal RAAS activation, hypertension in HIV infected individuals may include: microbial translocation, chronic inflammation, immune suppression and reconstitution, viral tropism, lipodystrophy, adipokines, and HIVrelated renal disease [91]. In the event of HIV infection, pregnant women's adherence to ART increases the risk of pre-term birth (PTB) [95]. Kenyan researchers were able to show that among preterm placentae, HIV infection was significantly associated with thrombosis, infarction, anomalies in cord insertion, gross evidence of membrane infection, and reduced placental thickness. Overall, preterm placentas in both HIV infected and HIV uninfected groups were associated with immature villi, syncytial knotting, villitis, and deciduitis. Features of HIV infected versus HIV uninfected placentas included significant fibrinoid deposition with villus degeneration, syncytiotrophoblast delamination, red blood cell adhesion, hyper-vascularity, and reduction in both surface area and perimeter of the terminal villi. These results imply that HIV infection and/or ART are associated with morphological changes in preterm placentas that contribute to delivery before 37 weeks. Hyper-vascularity suggests that the observed pathologies may be attributable, in part, to hypoxia [95].

\section{Renin-Angiotensin-Aldosterone System and COVID-19}

Of interest, SARS-CoV-2 infects target host cells by binding to a single-pass type I membrane protein ACE2. This is facilitated by the spike (S) protein priming performed by the type II transmembrane serine proteases (TMPRSS2) [96]. The ACE2 is an aminopeptidase enzyme that has a physiological role in degrading substances including angiotensin II, a key protective mechanism in regulating blood pressure, vascular and heart functions. It is found in most organs such as heart, lung, kidney, vessels, brain, and others, including the placenta [97]. Histopathology studies of COVID-19 positive pregnancies have shown that ACE2 membranous expression occurs in the syncytiotrophoblast (ST), cytotrophoblasts, decidual perivascular cells, and endothelial and vascular smooth muscle cells of the chorionic villi [96]. The interlinkage between SARS-CoV-2 and ACE2 is not simply a virus-receptor interaction, as numerous pre-existing comorbidities originate from RAAS imbalance, thereby causing higher susceptibility to virus transmission or an increase in mortality risk after infection. Cardiovascular injury is associated with greater frequency of death and following a SARS-CoV-2 infection, the virus 
exacerbates the clinical condition because of its cytotoxicity, uncontrolled inflammatory response and RAAS homeostatic loss [98]. Of note, there had been a $30 \%$ increase in maternal deaths during the Covid-19 lockdown (2020) in SA compared with the same period in 2019 [99].

\section{Summary}

The AT1, AT2, and AT4 receptors are crucial in the regulation of the RAAS. The AT1 receptor is expressed across all trimesters of pregnancy, within placental tissue, eliciting vasoconstriction. The increased expression of AT1 receptor further supports the hypothesis that a tissue-based RAAS exists. This increased expression is associated with the severity of $\mathrm{PIH}$, implying that the increased expression may be involved in the pathogenesis of PE. In the uterine placental bed of normotensive pregnancies, AT2 receptor expression was shown to be lower as compared to non-pregnant subjects. In PE uterine placental bed, AT2 receptor demonstrated predominance as a receptor subtype. These findings imply that the maternal uterine placental bed plays an endocrine role by causing adjacent placental vasoconstriction of fetal chorionic villi vessels. This may progress to decreased maternal-fetal oxygen and nutrition exchange, a known characteristic of PE. The AT4 receptors have been found in extravillous trophoblast cells where they facilitate vasodilation. However, AT4R in placentae of PE was found to be significantly reduced compared to normotensive pregnancies, which showed high levels throughout pregnancy.

Information on these cascading pathways and interactions of RAAS receptors in HIV infected preeclamptic women is lacking especially in SA.

\section{Conclusion}

Unraveling the pathophysiology of PE remains contentious; a mystery in particular a multifactorial clinical state that adversely affects almost all vital organs of pregnant women. Understanding the crosstalk of RAAS and its receptors at the maternal fetal interface and the placental bed is important in the control of hypertension and warrants urgent investigation. In light of the high prevalence of PE and HIV infection globally, the control of RAAS at circulating and tissue level may enable pharmacological intervention to control blood pressure in preeclampsia thereby preventing harm to maternal and fetal well-being.

\section{Declarations}

Conflict of Interest The authors declare no conflicts of interest relevant to this manuscript.

Human and Animal Rights and Informed Consent This article does not contain any studies with human or animal subjects performed by any of the authors.

\section{References}

Papers of particular interest, published recently, have been highlighted as:

- Of importance

•- Of major importance

1. Fyhrquist F, Saijonmaa O. Renin-angiotensin system revisited. Journal of internal medicine. 2008;264(3):224-36.

2. Fountain JH, Lappin SL. Physiology, renin angiotensin system. 2017.

3. Patel S, Rauf A, Khan H, Abu-Izneid T. Renin-angiotensinaldosterone (RAAS): The ubiquitous system for homeostasis and pathologies. Biomedicine \& Pharmacotherapy. 2017;94:317-25.

4. Pacurari M, Kafoury R, Tchounwou PB, Ndebele K. The reninangiotensin-aldosterone system in vascular inflammation and remodeling. International journal of inflammation. 2014;2014:1-13.

5. Verdonk K, Visser W, Van Den Meiracker AH, Danser AJ. The renin-angiotensin-aldosterone system in pre-eclampsia: the delicate balance between good and bad. Clinical Science. 2014;126(8):537-44.

6. Kassebaum NJ, Bertozzi-Villa A, Coggeshall MS, Shackelford KA, Steiner C, Heuton KR, et al. Global, regional, and national levels and causes of maternal mortality during 1990-2013: a systematic analysis for the Global Burden of Disease Study 2013. The Lancet. 2014;384(9947):980-1004.

7. Peters A, Dubas CM, Lotfinejad N, Pittet D. Home deliveries in limited resource environments. American Journal of Infection Control. 2020;48(8):859-61.

8. Demographic SA. Health Survey (SADHS), 2016. Key indicators report 2016.

9. Ukah UV, De Silva DA, Payne B, Magee LA, Hutcheon JA, Brown $\mathrm{H}$, et al. Prediction of adverse maternal outcomes from preeclampsia and other hypertensive disorders of pregnancy: a systematic review. Pregnancy Hypertension. 2018;11:115-23.

10. Umesawa M, Kobashi G. Epidemiology of hypertensive disorders in pregnancy: prevalence, risk factors, predictors and prognosis. Hypertension Research. 2017;40(3):213-20.

11. Moodley J, Onyangunga O, Maharaj N. Hypertensive disorders in primigravid black South African women: a one-year descriptive analysis. Hypertension in Pregnancy. 2016;35(4):529-35.

12. Sidley P. AIDS is largest cause of maternal deaths in South Africa. BMJ. 2000;321(7274):1434.

13. Brown MA, Magee LA, Kenny LC, Karumanchi SA, McCarthy FP, Saito S, et al. Hypertensive disorders of pregnancy: ISSHP classification, diagnosis, and management recommendations for international practice. Hypertension. 2018;72(1):24-43.

14. Aouache R, Biquard L, Vaiman D, Miralles F. Oxidative stress in preeclampsia and placental diseases. International journal of molecular sciences. 2018;19(5):1496.

15. Brichant JF, Bonhomme V. Preeclampsia: an update. Acta Anaesthesiol Belg. 2014;65(4):137-49.

16. Africa SS. Mid-year population estimates. 2020.

17. Simbayi L, Zuma K, Zungu N, Moyo S, Marinda E, Jooste S, Mabaso M, Ramlagan S, North A, Van Zyl J. South African National HIV Prevalence, Incidence, Behaviour and 
Communication Survey, 2017: towards achieving the UNAIDS 9090-90 targets. 2019.

18. Moodley D, Moodley P, Sebitloane M, Soowamber D, McNaughton-Reyes HL, Groves AK, et al. High prevalence and incidence of asymptomatic sexually transmitted infections during pregnancy and postdelivery in KwaZulu Natal, South Africa. Sexually transmitted diseases. 2015;42(1):43-7.

19. Woldesenbet S, Kufa T, Lombard C, Manda S, Ayalew K, Cheyip M, et al. The 2017 national antenatal sentinel HIV survey. National Department of Health: South Africa; 2019.

20. Adams JW, Watts DH, Phelps BR. A systematic review of the effect of HIV infection and antiretroviral therapy on the risk of pre-eclampsia. International Journal of Gynecology \& Obstetrics. 2016;133(1):17-21.

21. Schweda F, Friis U, Wagner C, Skott O, Kurtz A. Renin release. Physiology. 2007;22(5):310-9.

22. Kaschina E, Steckelings UM, Unger T. Hypertension and the reninangiotensin-aldosterone system. Encyclopedia of Endocrine Diseases: Elsevier Editora; 2018. p. 505-10.

23. Coates D. The angiotensin converting enzyme (ACE). The international journal of biochemistry \& cell biology. 2003;35(6):769-73.

24. Keidar S, Kaplan M, Gamliel-Lazarovich A. ACE2 of the heart: from angiotensin I to angiotensin (1-7). Cardiovascular Research. 2007;73(3):463-9.

25. Chung O, Kühl H, Stoll M, Unger T. Physiological and pharmacological implications of AT1 versus AT2 receptors. Kidney International. 1998;54:S95-S9.

26. Allen AM, Zhuo J, Mendelsohn FA. Localization and function of angiotensin AT1 receptors. American Journal of Hypertension. 2000;13(S1):31S-8S.

27. Kawai T, Forrester SJ, O’Brien S, Baggett A, Rizzo V, Eguchi S. AT1 receptor signaling pathways in the cardiovascular system. Pharmacological research. 2017;125:4-13.

28. Nickenig G. Central role of the AT 1-receptor in atherosclerosis. Journal of Human Hypertension. 2002;16(3):S26-33.

29. Fanelli C, Zatz R. Linking oxidative stress, the renin-angiotensin system, and hypertension. Am Heart Assoc; 2011.

30. Zhu YC, Zhu YZ, Lu N, Wang MJ, Wang YX, Yao T. Role of angiotensin AT1 and AT2 receptors in cardiac hypertrophy and cardiac remodelling. Clinical and Experimental Pharmacology and Physiology. 2003;30(12):911-8.

31. Bucher M, Ittner K-P, Hobbhahn J, Taeger K, Kurtz A. Downregulation of angiotensin II type 1 receptors during sepsis. Hypertension. 2001;38(2):177-82.

32. Garrido AM, Griendling KK. NADPH oxidases and angiotensin II receptor signaling. Molecular and Cellular Endocrinology. 2009;302(2):148-58.

33. Takeda H, Katagata Y, Hozumi Y, Kondo S. Effects of angiotensin II receptor signaling during skin wound healing. The American Journal of Pathology. 2004;165(5):1653-62.

34. Cooper A, Robinson G, Vinson G, Cheung W, Pipkin FB. The localization and expression of the renin-angiotensin system in the human placenta throughout pregnancy. Placenta. 1999;20(5-6): 467-74.

35. Thapa L, He C, Chen H. Study on the expression of angiotensin II (ANG II) receptor subtype 1 (AT1R) in the placenta of pregnancyinduced hypertension. Placenta. 2004;25(7):637-41.

36. Seki H. The role of the renin-angiotensin system in the pathogenesis of preeclampsia-new insights into the renin-angiotensin system in preeclampsia. Medical hypotheses. 2014;82(3):362-7.

37. Hatip-Al-Khatib I, Hatip F, Matsunaga Y. The protective tributary angiotensin members of renin-angiotensin system display beneficial effects in the central nervous system disorders. Am J Pharmacol. 2018;1(1):1001.

38. Carey RM. Update on the role of the AT2 receptor. Current opinion in nephrology and hypertension. 2005;14(1):67-71.
39. Sandberg K, Ji H, Clark A, Shapira H, Catt KJ. Cloning and expression of a novel angiotensin II receptor subtype. Journal of Biological Chemistry. 1992;267(14):9455-8.

40. Vanderheyden PM. From angiotensin IV binding site to AT4 receptor. Molecular and Cellular Endocrinology. 2009;302(2): 159-66.

41. Singh KD, Karnik SS. Angiotensin receptors: structure, function, signaling and clinical applications. Journal of Cell Signaling. 2016;1(2).

42. Wright JW, Yamamoto BJ, Harding JW. Angiotensin receptor subtype mediated physiologies and behaviors: new discoveries and clinical targets. Progress in Neurobiology. 2008;84(2):157-81.

43.• Williams P, Mistry H, Innes B, Bulmer J, Pipkin FB. Expression of AT1R, AT2R and AT4R and their roles in extravillous trophoblast invasion in the human. Placenta. 2010;31(5):448-55 This article has characterised expression of AT4R and AT1R and AT2R in human placenta throughout normal pregnancy and in PE.

44.•• Khaliq OP, Konoshita T, Moodley J, Naicker T. Soluble angiotensin IV receptor levels in preeclampsia: is there a variation? The Journal of Maternal-Fetal \& Neonatal Medicine. 2020:1-6 This article measured serum $A T 4 R$ in women with $P E$.

45. Santos RA, e Silva ACS, Maric C, Silva DM, Machado RP, de Buhr I, et al. Angiotensin-(1-7) is an endogenous ligand for the $G$ protein-coupled receptor Mas. Proceedings of the National Academy of Sciences. 2003;100(14):8258-63.

46. Santos RAS, Sampaio WO, Alzamora AC, Motta-Santos D, Alenina N, Bader M, Campagnole-Santos MJ. The ACE2/angiotensin-(1-7)/MAS axis of the renin-angiotensin system: focus on angiotensin-(1-7). Physiol Rev. 2017.

47. Bader M, Alenina N, Young D, Santos RA, Touyz RM. The meaning of Mas. Hypertension. 2018;72(5):1072-5.

48. Gaspari TA, Vinh A, Jones ES, Widdop RE. Ganging up on angiotensin II type 1 receptors in vascular remodeling. Hypertension. 2012;60(1):17-9.

49. Iwai M, Horiuchi M. Devil and angel in the renin-angiotensin system: ACE-angiotensin II-AT1 receptor axis vs. ACE2-angiotensin-(1-7)-Mas receptor axis. Hypertension Research. 2009;32(7): 533-6.

50. Lubel J, Garg M. Renin-angiotensin-aldosterone system inhibitors in Covid-19. N Engl J Med. 2020;382:e92.

51. Irani RA, Xia Y. The functional role of the renin-angiotensin system in pregnancy and preeclampsia. Placenta. 2008;29(9):763-71 This review details the functional role of the RAAS during pregnancy and the impact of AT1-AA on PE.

52. Merrill DC, Karoly M, Chen K, Ferrario CM, Brosnihan KB. Angiotensin-(1-7) in normal and preeclamptic pregnancy. Endocrine. 2002;18(3):239-45.

53. Brosnihan KB, Merrill DC, Yamaleyeva LM, Chen K, Neves L, Joyner J, et al. Longitudinal study of angiotensin peptides in normal and pre-eclamptic pregnancy. Endocrine. 2020;69(2):410-9.

54. Nielsen AH, Schauser K, Poulsen K. Current topic: the uteroplacental renin-angiotensin system. Placenta. 2000;21(5-6): 468-77.

55.• Wiegel RE, Jan Danser A, Steegers-Theunissen RP, Laven JS, Willemsen SP, Baker VL, et al. Determinants of maternal reninangiotensin-aldosterone-system activation in early pregnancy: insights from 2 cohorts, The Journal of Clinical Endocrinology \& Metabolism. 2020;105(11):3505-17 This article investigates maternal factors that may activate RAAS in early pregnancies.

56.• Moodley J. Impact of HIV on the incidence of pre-eclampsia. Cardiovascular journal of Africa. 2013;24(2):5 This article discusses the role of HIV in the pathogenesis of PE.

57. Brosens JJ, Pijnenborg R, Brosens IA. The myometrial junctional zone spiral arteries in normal and abnormal pregnancies: a review of the literature. American journal of obstetrics and gynecology. 2002;187(5):1416-23. 
58. Anton L, Brosnihan KB. Systemic and uteroplacental reninangiotensin system in normal and pre-eclamptic pregnancies. Therapeutic advances in cardiovascular disease. 2008;2(5):34962 This review summarizes the key findings in the circulating and uteroplacental RAS in normal and pre-eclamptic pregnancies.

59. Herse F, Dechend R, Harsem NK, Wallukat G, Janke Jr, Qadri F, et al. Dysregulation of the circulating and tissue-based renin-angiotensin system in preeclampsia. Hypertension. 2007;49(3):604-11 This article interrogates multiple compounds of circulating and tissue-based RAAS in normal and PE pregnancies.

60.• Anton L, Merrill DC, Neves LA, Stovall K, Gallagher PE, Diz DI, et al. Activation of local chorionic villi angiotensin II levels but not angiotensin (1-7) in preeclampsia. Hypertension. 2008;51(4):106672 This article analyzed RAAS factors expressed in the chorionic villous of normotensive and preeclamptic pregnancies.

61. Anton L, Merrill DC, Neves LA, Diz DI, Corthorn J, Valdes G, et al. The uterine placental bed renin-angiotensin system in normal and preeclamptic pregnancy. Endocrinology. 2009;150(9):431625.

62. Aung M, Konoshita T, Moodley J, Gathiram P. Association of gene polymorphisms of four components of renin-angiotensinaldosterone system and preeclampsia in South African black women. European Journal of Obstetrics \& Gynecology and Reproductive Biology. 2017;215:180-7 This article analyzed the distribution of gene polymorphisms in the RAAS that maybe associated with pathogenesis of $P E$.

63. Furuya M, Okuda M, Usui H, Takenouchi T, Kami D, Nozawa A, et al. Expression of angiotensin II receptor-like 1 in the placentas of pregnancy-induced hypertension. International Journal of Gynecological paThology. 2012;31(3):227-35 This article investigated the expression of angiotensin II receptor-like 1 (APJ) in human placentas with associated implications on fetal development.

64.• Delforce SJ, Lumbers ER, Ellery SJ, Murthi P, Pringle KG. Dysregulation of the placental renin-angiotensin system in human fetal growth restriction. Reproduction. 2019;158(3):237-45 This article investigated the mRNA expression and protein levels of RAAS components in placentae from pregnancies compromised by FGR.

65. Akbar SA, Khawaja NP, Brown PR, Tayyeb R, Bamfo J, Nicolaides KH. Angiotensin II type 1 and 2 receptors gene polymorphisms in pre-eclampsia and normal pregnancy in three different populations. Acta Obstetricia et Gynecologica Scandinavica. 2009;88(5):606-11.

66. Tanaka M, Jaamaa G, Kaiser M, Hills E, Soim A, Zhu M, et al. Racial disparity in hypertensive disorders of pregnancy in New York State: a 10-year longitudinal population-based study. American Journal of Public Health. 2007;97(1):163-70.

67. Wallukat G, Homuth V, Fischer T, Lindschau C, Horstkamp B, Jüpner A, et al. Patients with preeclampsia develop agonistic autoantibodies against the angiotensin AT 1 receptor. The Journal of Clinical Investigation. 1999;103(7):945-52.

68. LaMarca B, Parrish MR, Wallace K. Agonistic autoantibodies to the angiotensin II type I receptor cause pathophysiologic characteristics of preeclampsia. Gender Medicine. 2012;9(3):139-46.

69. Parrish MR, Ryan MJ, Glover P, Brewer J, Ray L, Dechend R, et al. Angiotensin II type 1 autoantibody induced hypertension during pregnancy is associated with renal endothelial dysfunction. Gender Medicine. 2011;8(3):184-8.

70. Dechend R, Viedt C, Müller DN, Ugele B, Brandes RP, Wallukat $\mathrm{G}$, et al. AT1 receptor agonistic antibodies from preeclamptic patients stimulate NADPH oxidase. Circulation. 2003;107(12): 1632-9.

71. Yia Y, Wne H, Bobst S, Day M-C, Kellems RE. Maternal autoantibodies from preeclamptic patients active angiotensin receptors on human trophoblast cells. Journal of the Society for Gynecologic Investigation. 2003;10(2):82-93.

72. Bobst SM, Day M-C, Gilstrap LC, Xia Y, Kellems RE. Maternal autoantibodies from preeclamptic patients activate angiotensin receptors on human mesangial cells and induce interleukin- 6 and plasminogen activator inhibitor-1 secretion. American Journal of Hypertension. 2005;18(3):330-6.

73. Yang L, Zhang S, Zheng R, Yan L, Zhao R, Liu H. Autoantibody against AT1 receptor from preeclamptic patients induces rats' endothelial injury. The FASEB Journal. 2009;23:812.14-4.

74. Yang X, Wang F, Chang H, Zhang S, Yang L, Wang X, et al. Autoantibody against AT1 receptor from preeclamptic patients induces vasoconstriction through angiotensin receptor activation. Journal of Hypertension. 2008;26(8):1629-35.

75. Trogstad L, Magnus P, Stoltenberg C. Pre-eclampsia: risk factors and causal models. Best practice \& research Clinical obstetrics \& gynaecology. 2011;25(3):329-42.

76. Sansone M, Sarno L, Saccone G, Berghella V, Maruotti GM, Migliucci A, et al. Risk of preeclampsia in human immunodeficiency virus-infected pregnant women. Obstetrics \& Gynecology. 2016;127(6):1027-32.

77. Powis KM, McElrath TF, Hughes MD, Ogwu A, Souda S, Datwyler SA, et al. High viral load and elevated angiogenic markers associated with increased risk of preeclampsia among Women Initiating Highly Active Antiretroviral Therapy (HAART) in Pregnancy in the Mma Bana Study, Botswana. Journal of Acquired Immune Deficiency Syndromes (1999). 2013;62(5):517.

78. Boyajian T, Shah PS, Murphy KE. Risk of preeclampsia in HIVpositive pregnant women receiving HAART: a matched cohort study. Journal of Obstetrics and Gynaecology Canada. 2012;34(2):136-41

79. Africa S, Health dDo. The 2013 National Antenatal Sentinel HIV Prevalence Survey South Africa. South Africa National Department of Health Pretoria; 2015.

80. Kalumba V, Moodley J, Naidoo T. Is the prevalence of preeclampsia affected by HIV/AIDS? A retrospective case-control study. Cardiovascular journal of Africa. 2013;24(2):24-7.

81. Hall D, Gebhardt S, Theron G, Grové D. Pre-eclampsia and gestational hypertension are less common in HIV infected women. Pregnancy Hypertension: An International Journal of Women's Cardiovascular Health. 2014;4(1):91-6.

82. Padayachee S, Moodley J, Naicker T. A review of angiogenic imbalance in HIV-infected hypertensive disorders of pregnancy. Current Hypertension Reports. 2019;21(9):1-11.

83. Naidoo G, Khaliq OP, Moodley J, Naicker T. The role of soluble Eselectin in HIV associated preeclampsia. European Journal of Obstetrics \& Gynecology and Reproductive Biology. 2021;257: 64-9.

84. David M, Moodley J, Naicker T. The function of adipsin and C9 protein in the complement system in HIV-associated preeclampsia. Archives of gynecology and obstetrics. 2021:1-7.

85. Landi B, Bezzeccheri V, Guerra B, Piemontese M, Cervi F, Cecchi $\mathrm{L}$, et al. HIV infection in pregnancy and the risk of gestational hypertension and preeclampsia. World Journal of Cardiovascular Diseases. 2014;2014.

86. Ekubagewargies DT, Kassie DG, Takele WW. Maternal HIV infection and preeclampsia increased risk of low birth weight among newborns delivered at University of Gondar specialized referral hospital, Northwest Ethiopia, 2017. Italian Journal of Pediatrics. 2019;45(1):1-7.

87. Frank KA, Buchmann EJ, Schackis RC. Does human immunodeficiency virus infection protect against preeclampsia-eclampsia? Obstetrics \& Gynecology. 2004;104(2):238-42. 
88. Tooke L, Riemer L, Matjila M, Harrison M. Antiretrovirals causing severe pre-eclampsia. Pregnancy Hypertension: An International Journal of Women's Cardiovascular Health. 2016;6(4):266-8.

89. Sebitloane HM, Moodley J, Sartorius B. Associations between HIV, highly active anti-retroviral therapy, and hypertensive disorders of pregnancy among maternal deaths in South Africa 20112013. International Journal of Gynecology \& Obstetrics. 2017;136(2):195-9.

90. Phoswa WN, Naicker T, Ramsuran V, Moodley J. Pre-eclampsia: the role of highly active antiretroviral therapy and immune markers. Inflammation Research. 2019;68(1):47-57.

91. Fahme SA, Bloomfield GS, Peck R. Hypertension in HIV-infected adults: novel pathophysiologic mechanisms. Hypertension. 2018;72(1):44-55.

92. Srinivasa S, Fitch KV, Wong K, Torriani M, Mayhew C, Stanley T, et al. RAAS activation is associated with visceral adiposity and insulin resistance among HIV-infected patients. The Journal of Clinical Endocrinology \& Metabolism. 2015;100(8):2873-82.

93. Chandel N, Ayasolla K, Lan X, Rai P, Mikulak J, Husain M, et al. Renin modulates HIV replication in T cells. Journal of Leukocyte Biology. 2014;96(4):601-9.

94. Tzoupis H, Leonis G, Megariotis G, Supuran CT, Mavromoustakos T, Papadopoulos MG. Dual inhibitors for aspartic proteases HIV-1 PR and renin: advancements in AIDS-hypertension-diabetes linkage via molecular dynamics, inhibition assays, and binding free energy calculations. Journal of Medicinal Chemistry. 2012;55(12):5784-96.
95. Obimbo MM, Zhou Y, McMaster MT, Cohen CR, Qureshi Z, Ong'ech J, et al. Placental structure in preterm birth among HIVpositive versus HIV-negative women in Kenya. Journal of acquired immune deficiency syndromes (1999). 2019;80(1):94.

96. Wong YP, Khong TY, Tan GC. The Effects of COVID-19 on Placenta and Pregnancy: What Do We Know So Far? Diagnostics. 2021;11(1):94.

97. Liu M, Wang T, Zhou Y, Zhao Y, Zhang Y, Li J. Potential role of ACE2 in coronavirus disease 2019 (COVID-19) prevention and management. Journal of Translational Internal Medicine. 2020;8(1):9-19.

98. Aleksova A, Gagno G, Sinagra G, Beltrami AP, Janjusevic M, Ippolito G, et al. Effects of SARS-CoV-2 on cardiovascular system: the dual role of angiotensin-converting enzyme 2 (ACE2) as the virus receptor and homeostasis regulator-review. International Journal of Molecular Sciences. 2021;22(9):4526.

99. Soma-Pillay P, Moodley J, Pattinson R, Fawcus S, Gebhardt S, Niit $\mathrm{R}$, editors. The effect of the first wave of Covid-19 on use of maternal and reproductive health services and maternal deaths in South Africa. Obstetrics and Gynaecology Forum; 2020: In House Publications.

Publisher's Note Springer Nature remains neutral with regard to jurisdictional claims in published maps and institutional affiliations. 